\title{
Comparison of diagnostic values between ultrasound elastography and ultrasound-guided thyroid nodular puncture in thyroid nodules
}

\author{
XIAOJIE PAN and LEI WANG \\ Department of Ultrasound, Liaocheng People's Hospital, Liaocheng, Shandong 252000, P.R. China
}

Received April 16, 2018; Accepted July 24, 2018

DOI: $10.3892 / 01.2018 .9257$

\begin{abstract}
Diagnostic value between ultrasound elastography and ultrasound-guided fine needle puncture biopsy in thyroid nodules was compared to provide reference for the selection of thyroid nodule (TN) diagnostic mode. A total of 194 patients with TN, admitted from June 2014 to June 2015, were selected to be treated with ultrasound elastography and ultrasound-guided fine needle puncture biopsy. The ultrasonic elasticity score and results of ultrasound-guided TN puncture were compared with diagnostic results of surgical or ultrasound follow-up for 12 months, and the sensitivity, specificity and accuracy of differentiating and diagnosing $\mathrm{TN}$ of the two methods were calculated to compare the clinical diagnostic values. A total of 194 patients were diagnosed with TN 217, including 129 benign and 88 malignant lesions. All the nodules were obtained by effective ultrasound elastography and ultrasound-guided fine needle puncture cytological pathological specimens, and it was diagnosed by ultrasound elastography that there were 75 benign nodules and 142 malignant nodules. It was diagnosed by ultrasound-guided fine needle puncture that there were 112 benign, 78 malignant and 27 uncertain nodules. The specificity and accuracy of ultrasound-guided fine needle puncture in the diagnosis of $\mathrm{TN}$ were higher than that of ultrasound elastography and the differences were statistically significant $(\mathrm{P}<0.05)$, while there was no statistically significant difference between the sensitivity of ultrasound elastography and ultrasound-guided fine needle puncture biopsy in the diagnosis of TN $(\mathrm{P}>0.05)$. Ultrasound elastography in the diagnosis of TN has a low specificity and the diagnosis of ultrasound-guided fine needle puncture biopsy may have uncertain results. There are some defects in the two diagnostic schemes and their combined application
\end{abstract}

Correspondence to: Dr Lei Wang, Department of Ultrasound, Liaocheng People's Hospital, 67 Dongchang West Road, Liaocheng, Shandong 252000, P.R. China

E-mail:whz2qn@163.com

Key words: ultrasound elastography, ultrasound-guided fine needle puncture biopsy, thyroid nodule, diagnostic values can complement each other's advantages and improve the early diagnostic efficiency of TN.

\section{Introduction}

As the largest endocrine organ in the human body, thyroid plays an important role in regulating the metabolic process of human body (1). Thyroid nodule (TN), is the most common thyroid disease in clinical practice. $\mathrm{TN}$ is a disease caused by abnormal growth of thyroid follicles in patients, most of which are benign lesions such as goiter and thyroid adenoma, but TN can also lead to malignant lesions such as thyroid carcinoma and lymphoma $(2,3)$. It has been reported (4) that the main reason for the significant increase of the incidence of thyroid cancer in the past 30 years is that there are no obvious clinical manifestations before the onset of thyroid cancer and it is very easy to have missed diagnosis. After thyroidectomy, the impairment of body function is accompanied by a variety of complications, which has a serious impact on the quality of life and safety of patients (5). Therefore, the diagnosis and differentiation of $\mathrm{TN}$ is particularly important.

There was a survey showing (6) that the diagnosis rate of diagnosing TN patients was $<10 \%$ in clinical palpation, but the detection rate of $\mathrm{TN}$ patients could be significantly improved by imaging examination. At present, the diagnosis of $\mathrm{TN}$ is mainly ultrasound-guided fine needle puncture, but the hardness of lesion tissue cannot be obtained by ultrasound imaging, while ultrasound elastography makes up for this defect, and it is being popularized to clinic gradually $(7,8)$.

The present study aimed to find a better diagnostic method and to provide reference for clinical practice by comparing the difference between ultrasound-guided fine needle puncture and elastic ultrasound in the diagnosis of TN.

\section{Patients and methods}

In the present study, 194 patients with $\mathrm{TN}$, admitted from June 2014 to June 2015, were selected to be treated with ultrasound elastography and ultrasound-guided fine needle puncture biopsy and all patients had definite diagnosis after surgical or ultrasound follow-up for 12 months. Among the 194 patients, 79 were male and 115 were female, and the age of the patients was 29-64 years, with an average age of $44.95 \pm 6.82$ years. The benign nodules were 129 and the 
malignant nodules were 88 . The clinical data are shown in Table I. This study was approved by the Medical Ethics Committee of Liaocheng People's Hospital (Liaocheng, China) and all patients and their families were informed and signed the informed consent.

Inclusion and exclusion criteria. Inclusion criteria: The patient was older than 18 years. The pathological diagnosis of $\mathrm{TN}$ after operation was clear and the size and location of the lesion could be described in detail. The course of the disease was half a year, and there was no recent drug treatment and no other hereditary diseases.

Exclusion criteria: Respiratory diseases, blood relationship between patients, no recent blood transfusion treatment, uncoordinated follow-up and incomplete clinical information.

\section{Detection methods}

Ultrasound elastography. In this experiment, Hi Vision Ascendus (Hitachi color ultrasound diagnostic instrument; Hitachi, Ltd., Tokyo, Japan) was used to detect 194 patients, and real-time elastic ultrasonic detection was performed on patients with real-time linear array high-frequency probe, with a probe frequency of 6-13 MHz. The patient presented supine position and exposed the neck. The diameter, shape, perimeter, and blood supply of the thyroid gland were detected by conventional ultrasound. Then, ultrasound elastography was used to detect the lesion and the vertical pressure was placed at the lesion. The pressure was adjusted to the range of 3-4 and the obtained images were observed (9). The ratings are shown in Table II. In this study, the patients with 1 and 2 score of elasticity were classified as benign nodules and those with elastic score $>3$ were classified as malignant nodules (10).

Ultrasound-guided fine needle puncture. In the present study, 16G automatic biopsy needle was used for ultrasound-guided puncture performed on patients. The patient presented supine position and exposed the neck. Routine disinfection was done and towels were held. The fixed converter was adjusted and fixed, the lesion was placed in the center of ultrasound image and the direction of the needle was inclined along the scanning plane. When the puncture reached the location of the lesion, the tissue of the lesion was sucked and biopsy was carried out. Results of cytological test referred to the literature (11): Benign: cytological detection was benign; malignant: cytological detection was malignant; uncertain: uncertain, suspicious as malignant nodules. An image of ultrasound puncture is shown in Fig. 1.

Statistical method. In this experiment, SPSS 20.0 statistical software package (IBM Corp., Armonk, NY, USA) was used to analyze the collected data, and GraphPad software was used to draw the histogram. The sensitivity was equal to true malignancy divided by pathological diagnosis of malignancy, the specificity was equal to true benign divided by pathologic diagnosis of benign, and the accuracy was equal to true malignancy plus true benign and then divided by total number of nodules. The counting data were expressed by rate (\%), and analyzed by the Chi-square test. $\mathrm{P}<0.05$, was considered as statistically significant.
Table I. Clinical data of patients.

\begin{tabular}{lc}
\hline Groups & Clinical information (n) \\
\hline Sex & \\
Male & 79 \\
Female & 115 \\
Age (years) & \\
$>45$ & 105 \\
$\leq 45$ & 89 \\
Benign lesion (n=129) & \\
Adenomatous goiter & 82 \\
Follicular adenoma & 25 \\
Proliferative nodule & 22 \\
Malignant lesion (n=88) & \\
Papillary thyroid carcinoma & 81 \\
Follicular thyroid carcinoma & 6 \\
Medullary carcinoma & 1 \\
\hline
\end{tabular}

Table II. Elastic ultrasound ratings.

\begin{tabular}{ll}
\hline Ratings & \multicolumn{1}{c}{ Standards } \\
\hline 1 point & $\begin{array}{l}\text { The nodules and surrounding tissues are green } \\
\text { The nodules were mixed with blue and green, } \\
\text { but mostly green } \\
\text { The nodules were mixed with blue and green, } \\
\text { but mostly blue }\end{array}$ \\
4 points & $\begin{array}{l}\text { The nodule is blue } \\
\text { The nodules and surrounding tissues are blue }\end{array}$ \\
\hline
\end{tabular}

\section{Results}

Diagnostic results of the two methods. In this study, 194 patients were detected and it was found that the number of nodules diagnosed through surgical or ultrasound follow-up for 12 months was 217, including 129 benign nodules and 88 malignant nodules. Through ultrasound elastography, it was diagnosed that there were 75 benign nodules and 142 malignant nodules, and through ultrasound-guided puncture, it was diagnosed that there were 112 benign nodules, 78 malignant nodules and 27 uncertain nodules (Tables III and IV).

Comparison of diagnostic values of the two methods. Through the calculation of the sensitivity, specificity and accuracy by the two detection methods, it was found that there was no statistically significant difference $(P>0.05)$ between the sensitivity of ultrasound elastography $(69.32 \%)$ and that of ultrasound-guided fine needle puncture $(64.77 \%)$. By contrasting the specificity of ultrasound elastography, it was found that there was a significant difference $(\mathrm{P}<0.05)$ between the specificity of ultrasound elastography $(20.93 \%)$ and that of ultrasound-guided fine needle puncture (83.7\%). Besides, through the calculation of accuracy, it was found that the accuracy of ultrasound elastography $(40.55 \%)$ was significantly 

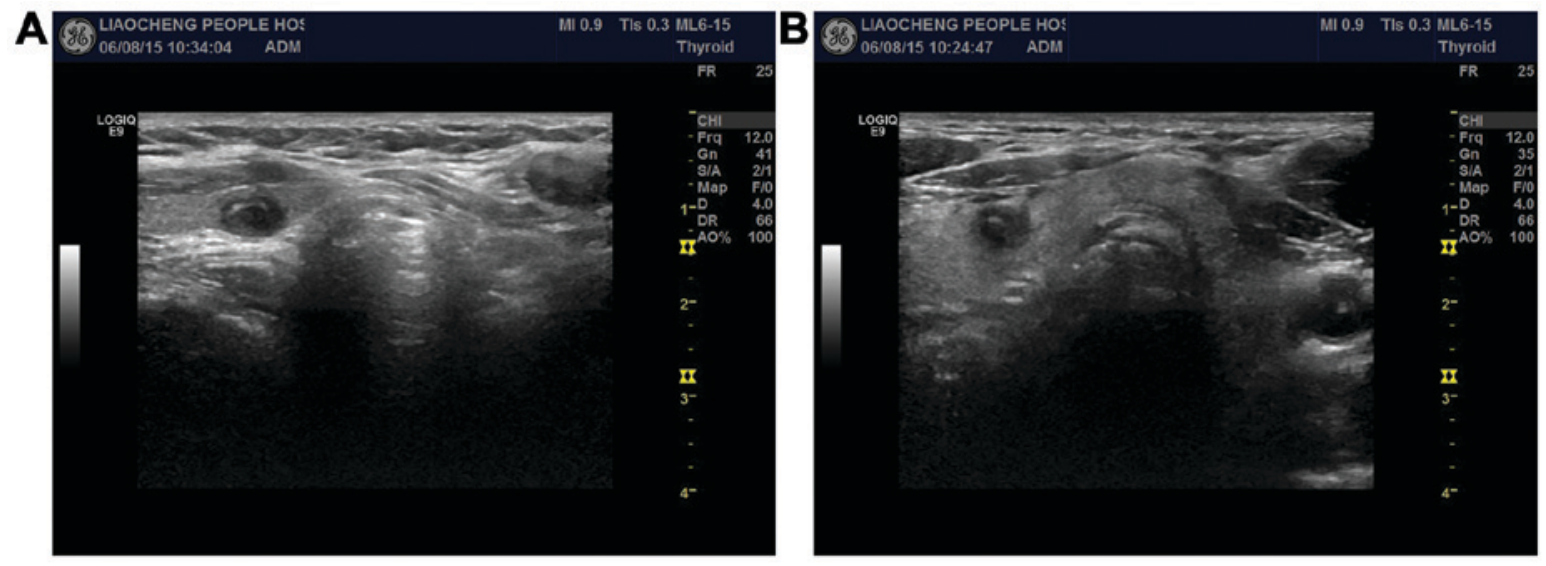

Figure 1. Image of ultrasound puncture. (A) The right lobe of the thyroid is a solid nodule with arc calcification around it. (B) A cross-sectional display of nodules.

Table III. Results of ultrasound imaging.

\begin{tabular}{lccc}
\hline & \multicolumn{2}{c}{$\begin{array}{c}\text { Results of } \\
\text { ultrasonic diagnosis }\end{array}$} \\
\cline { 2 - 3 } $\begin{array}{l}\text { Results of } \\
\text { pathological diagnosis }\end{array}$ & Malignant & Benign & Total \\
\hline Malignant & 61 & 48 & 109 \\
Benign & 81 & 27 & 108 \\
Total & 142 & 75 & 217 \\
\hline
\end{tabular}

Table IV. Results of ultrasound-guided puncture.

\begin{tabular}{lcccr}
\hline & \multicolumn{3}{c}{$\begin{array}{l}\text { Results of } \\
\text { Results of } \\
\text { pathological } \\
\text { diagnosis }\end{array}$} & \multicolumn{3}{c}{ ultrasound-guided puncture } \\
\cline { 2 - 4 } & Malignant & Benign & Uncertain & Total \\
\hline Malignant & 57 & 4 & 27 & 88 \\
Benign & 21 & 108 & 0 & 129 \\
Total & 78 & 112 & 27 & 217 \\
\hline
\end{tabular}

lower than that of ultrasound-guided fine needle puncture (76.04\%; P<0.05) (Fig. 2).

\section{Discussion}

Nowadays, TN is a common endocrine system disease in clinic. There was a survey showing that the incidence of TN was only $4-8 \%$ and the incidence of malignant lesions was $<1 \%$, while the diagnostic rate of TN was very unsatisfactory (12). Statistics showed (13) that the diagnostic rate of TN before clinical operation was $\leq 50 \%$, and most of the patients were found to be middle and late stage after diagnosis, which brought great difficulties to the treatment and prognosis of the patients. Therefore, the early diagnosis of TN plays a very important guiding role in clinical treatment. At present, with the increasing awareness of self-protection and safety of the masses, a large number of people conduct regular physical

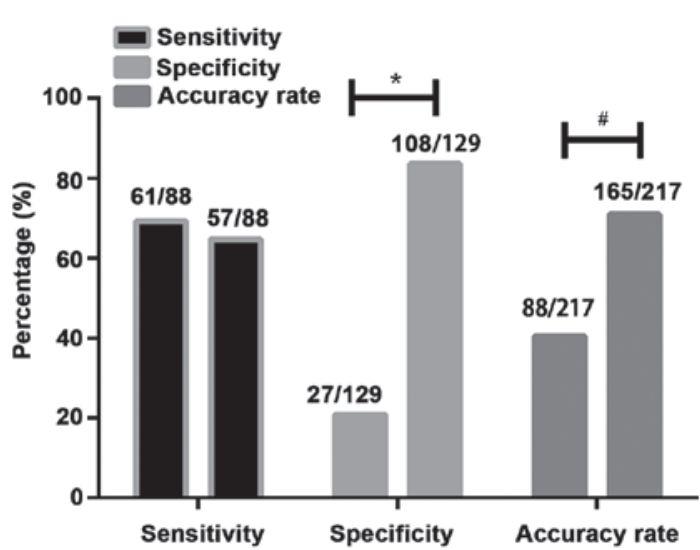

Figure 2. Comparison of diagnostic values between ultrasound elastography and ultrasound-guided fine needle puncture. By contrasting the sensitivity, specificity and accuracy of ultrasound elastography and ultrasound-guided fine needle puncture, it was found that the specificity and accuracy of ultrasound-guided fine needle puncture were significantly higher than that of ultrasound elastography, and the differences were statistically significant $(\mathrm{P}<0.05) .{ }^{*} \mathrm{P}<0.05,{ }^{\#} \mathrm{P}<0.01$.

examination every year, and thyroid detection has been paid more and more attention.

The diagnosis of TN is mainly based on ultrasound and palpation puncture and the advantages and disadvantages of palpation puncture are obvious. The failure rate of puncture is high and it is difficult to obtain materials for degenerative and small nodules, which leads to the failure of the later examination (14). The traditional grayscale supergrowth and color Doppler ultrasound have a good judgment on the tissue morphology, the perimeter, the internal echo of the tissue, the blood flow in and around the tissue and the calcification of the tissue, but the hardness of the tissue cannot be judged very well (15). Ultrasound elastography is an ultrasound imaging method first proposed by scholar Ophir et al (16). Ultrasound elastography is to compare the difference of elastic coefficient between different tissues and after compression by external force, the tissue changes in different forms. The amplitude of signal movement before and after compression is contrasted and transformed into a real-time image, so as to judge the hardness of the tissue through it. The greater the hardness is, the higher the degree of malignancy is, and vice versa (17). 
In this study, through the diagnosis of TN patients by ultrasound elastography, it was found that the sensitivity of ultrasound elastography was $69.32 \%$, which was slightly higher than that of ultrasound-guided puncture $(64.77 \%)$, but there was no statistically significant difference. However, in the study of Li et al (18), the accuracy of detecting TN by ultrasound elastography was as high as $92.5 \%$ (74/80), which was very different from our study, and we speculated that it might be caused by the differences of equipment. Compared with ultrasound-guided puncture, the specificity and accuracy were significantly lower, which might be due to the internal hemorrhage, necrosis and calcification in some benign $\mathrm{TN}$, so that the hardness of tissue increased, resulting in a lot of false-positive results (19). When TN diameter was $>3 \mathrm{~cm}$ and at the lower pole position, the detection results would also be affected, leading to the diagnosis deviation (20). Compared with palpation-guided puncture biopsy, ultrasound-guided high-frequency puncture had obvious advantages. First of all, the depth and the orientation of the needle were more clearly grasped. Secondly, the ultrasound-guided puncture wound was smaller, faster and safer, and the failure rate could be reduced and the efficiency could be improved through ultrasound guidance (21). In this experiment, the results of ultrasound-guided puncture in the diagnosis of TN showed that the sensitivity, specificity and accuracy of puncture TN under ultrasound guidance were satisfactory and the specificity and accuracy were significantly higher than that of ultrasound elastography. Nevertheless, in the case of multiple advantages, ultrasound-guided puncture is still an invasive detection method. If the patient has small nodules and more lesions, it may result in limited safety during the examination. Moreover, the ultrasound-guided fine needle puncture will have a small number of sucked cells, and the small number of sucked cells will result in unsatisfactory results under post-staining microscope (22). We speculated that in this ultrasound-guided fine needle puncture, uncertain patients might be the result of a small number of sucked cells. Both methods had advantages and disadvantages. Through consulting the literature, it was found $(23,24)$ that the detection rate of TN could be improved by ultrasound elastography combined with ultrasound-guided puncture and the screening of patients by ultrasound elastography could reduce the number of puncture and false-positive results and increase the early diagnosis rate.

However, there are still some limitations in this study. First of all, this experiment is a retrospective analysis, lacking contrast. Secondly, the sample size is small, which may be one of the reasons for the deviation of the results of this study. Therefore, we hope to increase the number of samples in future studies and to establish a randomized controlled trial to verify the correctness of the results of this study.

In summary, ultrasound elastography and ultrasound-guided fine needle puncture biopsy may lead to different results, and there are some limitations in a single diagnostic scheme. Therefore, the two diagnostic schemes need to be combined to complement each other so as to improve the early diagnosis of TN.

\section{Acknowledgements}

Not applicable.

\section{Funding}

No funding was received.

\section{Availability of data and materials}

The datasets used and/or analyzed during the present study are available from the corresponding author on reasonable request.

\section{Authors' contributions}

XP drafted the manuscript. XP and LW performed the ultrasound elastography and needle puncture. Both authors read and approved the final manuscript.

\section{Ethics approval and consent to participate}

The study was approved by the Ethics Committee of Liaocheng People's Hospital (Liaocheng, China). Signed informed consents were obtained from the patients or the guardians.

\section{Patient consent for publication}

Not applicable.

\section{Competing interests}

The authors declare that they have no competing interests.

\section{References}

1. Tuttle RM, Haugen B and Perrier ND: Updated American Joint Committee on Cancer/tumor-node-metastasis staging system for differentiated and anaplastic thyroid cancer (eighth edition): What changed and why? Thyroid 27: 751-756, 2017.

2. Parsa AA and Gharib H: History and examination for thyroid nodules. In: Thyroid Nodules. Gharib H (ed). 1st edition. Humana Press, Cham, pp13-18, 2018.

3. Alexander EK, Kennedy GC, Baloch ZW, Cibas ES, Chudova D, Diggans J, Friedman L, Kloos RT, LiVolsi VA, Mandel SJ, et al: Preoperative diagnosis of benign thyroid nodules with indeterminate cytology. N Engl J Med 367: 705-715, 2012.

4. Lim H, Devesa SS, Sosa JA, Check D and Kitahara CM: Trends in thyroid cancer incidence and mortality in the United States, 1974-2013. JAMA 317: 1338-1348, 2017.

5. Lee JC, Grodski S, Yeung M and Serpell J: Response to the letter to the editor regarding 'Quantitative study of voice dysfunction after thyroidectomy'. Surgery 162: 692-693, 2017.

6. Paschke R, Cantara S, Crescenzi A, Jarzab B, Musholt TJ and Sobrinho Simoes M: European Thyroid Association guidelines regarding thyroid nodule molecular fine-needle aspiration cytology diagnostics. Eur Thyroid J 6: 115-129, 2017.

7. Middleton WD, Teefey SA, Reading CC, Langer JE, Beland MD, Szabunio MM and Desser TS: Multiinstitutional analysis of thyroid nodule risk stratification using the American College of Radiology Thyroid Imaging Reporting and Data System. AJR Am J Roentgenol 208: 1331-1341, 2017.

8. Seo H,Na DG, Kim JH, Kim KW and Yoon JW: Ultrasound-based risk stratification for malignancy in thyroid nodules: A four-tier categorization system. Eur Radiol 25: 2153-2162, 2015.

9. Bae JM, Hahn SY, Shin JH and Ko EY: Inter-exam agreement and diagnostic performance of the Korean thyroid imaging reporting and data system for thyroid nodule assessment: Real-time versus static ultrasonography. Eur J Radiol 98: 14-19, 2018.

10. Hong Y, Liu X, Li Z, Zhang X, Chen M and Luo Z: Real-time ultrasound elastography in the differential diagnosis of benign and malignant thyroid nodules. J Ultrasound Med 28: 861-867, 2009. 
11. Singh Ospina N, Maraka S, Espinosa DeYcaza A, O'Keeffe D, Brito JP, Gionfriddo MR, Castro MR, Morris JC, Erwin P and Montori VM: Diagnostic accuracy of thyroid nodule growth to predict malignancy in thyroid nodules with benign cytology: Systematic review and meta-analysis. Clin Endocrinol (Oxf) 85: $122-131,2016$

12. Lee YH, Baek JH, Jung SL, Kwak JY, Kim JH and Shin JH; Korean Society of Thyroid Radiology (KSThR); Korean Society of Radiology: Ultrasound-guided fine needle aspiration of thyroid nodules: A consensus statement by the Korean Society of Thyroid Radiology. Korean J Radiol 16: 391-401, 2015.

13. Haugen BR, Alexander EK, Bible KC, Doherty GM, Mandel SJ, Nikiforov YE, Pacini F, Randolph GW, Sawka AM, Schlumberger M, et al: 2015 American Thyroid Association management guidelines for adult patients with thyroid nodules and differentiated thyroid cancer: The American Thyroid Association guidelines task force on thyroid nodules and differentiated thyroid cancer. Thyroid 26: 1-133, 2016.

14. Carneiro-Pla D: Thyroid nodule biopsy. In: Management of Thyroid Nodules and Differentiated Thyroid Cancer. Roman SA Sosa JA and Solórzano CC (eds). 1st edition. Springer International Publishing, Cham, pp47-58, 2017.

15. Ma JJ, Ding H, Xu BH, Xu C, Song LJ, Huang BJ and Wang WP. Diagnostic performances of various gray-scale, color Doppler, and contrast-enhanced ultrasonography findings in predicting malignant thyroid nodules. Thyroid 24: 355-363, 2014.

16. Ophir J, Céspedes I, Ponnekanti H, Yazdi Y and Li X: Elastography: A quantitative method for imaging the elasticity of biological tissues. Ultrason Imaging 13: 111-134, 1991.

17. Marticorena Garcia SR, Guo J, Dürr M, Denecke T, Hamm B, Sack I and Fischer T: Comparison of ultrasound shear wave elastography with magnetic resonance elastography and renal microvascular flow in the assessment of chronic renal allograft dysfunction. Acta Radiol: Jan 1, 2017 (Epub ahead of print). doi: 10.1177/0284185117748488.
18. Li F, Zhang J, Wang Y and Liu L: Clinical value of elasticity imaging and contrast-enhanced ultrasound in the diagnosis of papillary thyroid microcarcinoma. Oncol Lett 10: 1371-1377, 2015.

19. Russ G: Risk stratification of thyroid nodules on ultrasonography with the French TI-RADS: Description and reflections. Ultrasonography 35: 25-38, 2016.

20. Dudea SM and Botar-Jid C: Ultrasound elastography in thyroid disease. Med Ultrason 17: 74-96, 2015.

21. Levine RA and Interlandi J: The procedure of ultrasound-guided percutaneous biopsy of thyroid and cervical lymph nodes: Technical steps, pitfalls, and pearls. In: Advanced Thyroid and Parathyroid Ultrasound. Milas M, Mandel S and Langer JE (eds). 1st edition. Springer International Publishing, Cham, pp309-321, 2017.

22. Kim H, Kim JA, Son EJ and Youk JH: Quantitative assessment of shear-wave ultrasound elastography in thyroid nodules: Diagnostic performance for predicting malignancy. Eur Radiol 23: 2532-2537, 2013

23. Giovannini M, Thomas B, Erwan B, Christian P, Fabrice C, Benjamin E, Geneviève M, Paolo A, Pierre D, Robert Y, et al: Endoscopic ultrasound elastography for evaluation of lymph nodes and pancreatic masses: A multicenter study. World J Gastroenterol 15: 1587-1593,2009.

24. Monpeyssen H, Tramalloni J, Poirée S, Hélénon O and Correas JM: Elastography of the thyroid. Diagn Interv Imaging 94: 535-544, 2013.

(c) () $\Theta$ This work is licensed under a Creative Commons EY NO NO Attribution-NonCommercial-NoDerivatives 4.0 International (CC BY-NC-ND 4.0) License. 